International Journal of Current Advanced Research

ISSN: O: 2319-6475, ISSN: P: 2319 - 6505, Impact Factor: SJIF: 5.995

Available Online at www.journalijcar.org

Volume 6; Issue 4; April 2017; Page No. 3150-3152

DOI: http://dx.doi.org/10.24327/ijcar.2017.3152.0207

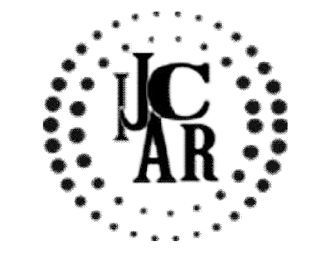

Review Article

\title{
CURVED CANAL-MISHAP AND MANAGEMENT- A REVIEW
}

\author{
Amurdhavani ${ }^{1}$ and M G Aarthy ${ }^{2}$
}

Saveetha Dental College and Hospital, Chennai

\begin{tabular}{l}
\hline A R T I C L E I N F O \\
\hline Article History: \\
Received $11^{\text {th }}$ January, 2017 \\
Received in revised form $19^{\text {th }}$ February, 2017 \\
Accepted $22^{\text {nd }}$ March, 2017 \\
Published online $28^{\text {th }}$ April, 2017
\end{tabular}

Key words:

Canal-Mishap, Management

\begin{abstract}
A B S T R A C T
Aim: The aim of this review is to focus on mishaps related to curved root canal and its managing methods.

Background: Nature seldom draws a straight line. Nowhere is this more apparent than in the anatomy of teeth roots and root canal systems of human teeth.Canal shaping is a critical aspect of endodontic treatment because it influences the outcome of the subsequent phases of canal irrigation and filling and the success of the treatment itself. In fact, curved canals are the most common endodontic complexity. The most frequent problems occurring in canal preparation are root perforation, ledging and instrument separation. These problems usually occur as a result of forcing and driving the instrument, over use of reaming action, and over reliance on chelating agents.

Reason: Concepts concerning the role and purpose of this canal preparation, however, have differed remarkably at different times in the development of endodontics and in the hands of different practitioners. Thus in this review we will discuss about mishaps related to curved canals and its management.
\end{abstract}

Copyright $₫ 2017$ Amurdhavani and M G Aarthy. This is an open access article distributed under the Creative Commons Attribution License, which permits unrestricted use, distribution, and reproduction in any medium, provided the original work is properly cited.

\section{INTRODUCTION}

A tooth with a straight root canal is an exception rather than being normal because most teeth show some curvature of the canal. The goal of quality endodontic therapy has remained the same from the beginning.Removal of pulpal tissues with proper cleaning and shaping, followed by an obturation system and coronal seal, will satisfy both mechanical and biological objectives.(1) As clinicians we need to appreciate each of these aspects and know that our therapy's success is dictated by the weakest element of our treatment. One area that has the potential for improvement is our ability to accurately instrument root canal systems in a manner that maintains the original path of curvature in both significant and multiple curvature systems. Presence of curvature may cause difficulty in root canal instrumentation. The final result of instrumentation of curved canals may be influenced by several factors such as flexibility and diameter of the endodontic instruments, instrumentation techniques, location of the foraminal opening and the hardness of dentin. Failing to notice canal curvature before treatment can lead to preparation errors such as apical zips, perforations, canal blockages, or instrument separation, which can leave the canal unprepared and lead to continued pathology compromising the outcome of treatment.(2) In the treatment of curved canals,proper instrumentation helps in achieving true success. These include small stainless steel hand files, nickel-titanium hand files, and rotary nickel titanium files.

*Corresponding author: Amurdhavani

Saveetha Dental College and Hospital, Chennai
Endodontic Mishaps

Endodontic mishaps are unfortunate occurrences that occurs during treatment. Some might be due to not noticing the details, whereas others are unpredictable (3). Failure to grasp the rationale behind cleaning and shaping concepts can increase the occurrence of needless complications such as blockages, ledge formation, apical transportation, and perforations. These have been attributed to the inappropriate cleaning and shaping concepts (4). Amongthecomplications most commonly observed during root canal instrumentation is a deviation from the original canal curvature without communicating with the periodontal ligament, resulting in a procedural error termed ledge formation. When the operator works the files short of the full canal length, and the canal becomes blocked at the apex (5). The presence of a ledge might exclude the possibility of achieving an adequately shaped canal preparation that reaches the ideal working length, and this can result in incomplete instrumentation and disinfection of the root canal system as well as incomplete filling of the canal. The root canal space apical to the ledgeis difficult to clean and shape thoroughly; therefore, ledges frequently result in periapical pathosis after the endodontic treatment. Consequently, there might be a causal relationship between ledge formation and unfavourable endodontic treatment outcomes $(4,6-11)$. Occasionally even very skilled and careful clinicians might create a ledge within a root canal when treating teeth with unsuspected aberrations in the anatomy of the canal. Because of the noticeable incidence of ledge formation in endodontic practice and the critical need for its recognition and management, this review aims to 
address the etiology, associated factors, recognition, prevention, prognosis, and the important considerations in the endodontic management of the ledge.

\section{Cause}

The condition is thought to be due to trauma during the period in which tooth is forming. The result is that the position of the calcified portion of the tooth is changed [12].

Presence of curvature may cause difficulty in root canal instrumentation. The final result of instrumentation of curved canals may be influenced by several factors such as flexibility and diameter of the endodontic instruments, instrumentation techniques, location of the foraminal opening and the hardness of dentin.[13]

Ledge formation, blockages, perforations and apical transportation are accidents that have been observed to occur after preparation of curved canals. Management of such curved canals is an endodontic challenge; so, for a successful therapy, a thorough knowledge of root and root canal morphology is required. Curved canals can be [14]

- Gradual curvature of the mesial canals in the apical third;

- $\quad$ Acute curvature in the apical third;

- Curvature throughout the canal;

- Dilacerated root canal and

- S-shaped root canal

\section{Various techniques used for management}

Traditionally, canal shaping has been achieved using ISOnormed, 0.02-tapered stainless steel instruments. Sizes above \#15 or \#20 become inflexible and have a tendency to straighten. When carried out in curved canals, such procedure often results in iatrogenic damage to the natural shape of the canal, particularly in its apical third, resulting in errors like ledge, elbow or zipping of the canal.[14] To avoid occurrence of such errors, even contact of the file to canal dentine should be there. But the file has the tendency to straighten up, and hence it is difficult to control removal of dentine along the entire length of file in push pull motion.

The above errors can be reduced by [15]

1. Decreasing the restoring force by means of which straight file apt to bend against the curved dentine surface and

2. Decreasing the length of the file which is aggressively cutting at a given span.

Decreasing the force can be done by the following.

\section{Precurving the file}

A precurved file traverses the curve better than a straight file. Precurving is done in two ways:

*Placing a gradual curve for the entire length of the file *Placing a sharp curve of nearly $45^{\circ}$ near the apical end of the instrument

\section{Extravagant use of smaller number files as they can follow canal curvature}

Because of their flexibility, they should be used until larger files are able to negotiate the canal without force.

\section{Use of intermediate size of files}

It allows smoother transition of the instrument sizes to cause smoother cutting in curved canals, e.g. cutting $1 \mathrm{~mm}$ of No. 15 file makes it No. 17 file as there is an increase of $0.02 \mathrm{~mm}$ of diameter per millimeter of length.

\section{Use of flexible files}

As they help in maintaining shape of the curve and avoid errors like ledge, elbow or zipping of the canal.

Decrease in length of actively cutting files is achieved by the following:

Anti-curvature filing.

Modifying cutting edges of the instrument by dulling the flute on outer surface of apical third and inner portion of middle third, which can be done by diamond file.

Changing canal preparation techniques, i.e. use of crowndown technique. Tendency to create narrow canal shapes minimizing access of irrigants and creating potential to allow debris to be pushed apically. Attempts at overcoming the deficiencies of these instruments resulted in a number of preparation techniques that aimed to reduce iatrogenic defects and produce canals with a more flared shape.[14]

\section{Advantage}

- Efficient and less prone to cause iatrogenic damage and maintains the instruments centrally within the root canal.

Extrusion of debris apically is also reduced, resulting in less postoperative pain.

\section{Motion of file in a root canal}

Enlargement of a straight canal does not pose particular problems. It could be done by a succession of inward and outward motions accompanied or not by rotary motions. However, curved canals are different where departure from the balanced force technique can result in damage of the canal wall. As it is impossible to ascertain on the basis of radiograph whether there is a curvature in a buccolingual plane or not, it is expedient to apply the balanced force technique as a general rule for the preparation of all root canals.[16]

Introduction of very flexible instruments made from nickel titanium alloy having a taper 2-6 times greater than the ISO standardized 0.02 files have revolutionarised the management of curved canal. They have the ability to pass around curves more readily. They are available as GT instruments and Protaper instruments (Dentsply). Both instruments appear to offer many advantages over traditional 0.02 taper hand instruments. Both instruments can be used in conjunction with or complementary to rotary instruments. Hand NiTi instruments can also be selected instead of rotary instruments in teeth with difficult canal anatomy like severe curvature in apical third and problematic handpiece access.[14]

The crown-down sequence of instrumentation has largely superseded the outdated step-back method. The advantages of this root canal preparation from crown to apex with early coronal flaring include [14]: 
- $\quad$ reduced coronal binding of instruments;

- less likelihood for a change in the working length measurement during preparation;

- less risk of inoculation of endodontic pathogens into the periradicular tissues;

- enhanced penetration of irrigant into the root canal system and

- less risk of extrusion of irrigant and debris.

\section{DISCUSSION}

One of the most important aspects of endodontic treatment is the cleaning and shaping of the root canals. The most desirable shape of the prepared canal is a progressive taper with the largest diameter at the coronal end and is narrowest at the apical constriction.[16] A progressive taper allows a greater degree of instrument and irrigant contact with the surfaces of the canal walls, thereby enhancing the effectiveness of cleaning.[13] However, the preparation of curved canals presents one of the greatest challenges in endodontics and is fraught with difficulties. Only the curvatures in mesio-distal plane can be seen on radiograph, though curvatures in the bucco-lingual plane are also evident in many teeth. Failure of root canal treatment in curved canals is mainly due to procedural errors like ledges, fractured instruments, canal blockage, zip and elbow creation.[13]. The case presented above were treated with hand instrumentation with $\mathrm{K}$ files.

\section{CONCLUSION}

It is important that a tooth with complex root canal morphology be treated properly the very first time, since endodontic treatment in such teeth is likely to fail if not treated carefully. Proper care and attention should be directed in radiographic assessment, access cavity preparation and exploration to negotiate extra and curved canals.

\section{References}

1. Schilder H. Cleaning and shaping the root canal. Dent Clin North Am 1974; 18:269-96.

2. Peters OA, Peters CI. Cleaning and shaping the root canal system. In: Cohen S, Hargreaves KM, eds. Pathways of the pulp. 9th ed. St. Louis: Mosby, Inc., 2006:290-357.
3. IngleJI, BaklandLK. Endodontics. 5th ed. London: BCDeckerInc, 2002; 412,482-9, 525-38, 695, 729, 769, 776-85.

4. CohenS, Burns RC. Pathways of the pulp. 8th ed. St Louis: Mosby, 2002:94, 242-52, 530, 870, 910-6.

5. NagyCD, BarthaK, BernathM, VerdesE, SzaboJ. The effect of root canal morphology on canal shape following instrumentation using different techniques. Int Endod J 1997; 30:133-40.

6. Kapalas A, Lambrianidis T. Factors associated with root canal ledging during instrumentation. Endod Dent Traumatol 2000; 16:229-31.

7. Harty FJ, Parkins BJ, Wengraf AM. Success rate in root canal therapy: a retrospective study of conventional cases. Br Dent J 1970; 128:65-70.

8. Namazikhah MS, Mokhlis HR, Alasmakh K. Comparison between a hand stainlesssteel $\mathrm{K}$ file and a rotary NiTi 0.04 taper. J Calif Dent Assoc 2000; 28:421-6.

9. Greene KJ, Krell KV. Clinical factors associated with ledged canals in maxillary and mandibular molars. Oral Surg Oral Med Oral Pathol 1990; 70:490-7.

10. Gutmann JL, Dumsha TC, Lovdahl PE, Hovland EJ. Problem solving in endodontics. 3rd ed. St Louis: Mosby, 1997:96-100, 117.

11. CohenS, HargreavesKM. Pathway softhepulp. 9thed.StLouis: Mosby, 2006:992-4.

12. Jafarzadeh H, Abbott PV. Dilaceration: review of an endodontic challenge. $J$ Endod. 2007;33:102530.[PubMed]

13. Hamasha AA, Al-Khateeb T, Darwazeh A. Prevalence of dilaceration in Jordanian adults. Int Endod J. 2002; 35:910-2. [PubMed]

14. Jain N, Tushar S. Curved canals: ancestral files revisited. Indian J Dent Res. 2008; 19:267-71. [PubMed]

15. Stock CR, Gulabiwala WK. Endodontics. 3th ed. St Louis: Mosby; 2004.

16. Charles TJ, Charles JE. The 'balanced force' concept for instrumentation of curved canals revisited. Int Endod J. 1998; 31:166-72. [PubMed]

\section{How to cite this article:}

Amurdhavani and M G Aarthy (2017) ' Curved Canal-Mishap And Management- A Review', International Journal of Current Advanced Research, 06(04), pp. 3150-3152.

DOI: http://dx.doi.org/10.24327/ijcar.2017.3152.0207 